day. The rate performed by minimal access surgery(MAS) increased from $33 \%$ to $93 \%$ despite an increasingly obese population.

Conclusion In 2019, 93\% of women treated in Guildford for endometrial cancer, received MAS. Since 2008 our conversion rate to open has fallen from $18 \%$ to $1.7 \%$, median EBL from $300 \mathrm{ml}$ to $50 \mathrm{ml}$ and our median LOS from 6 days to 1 night. In our experience, Robotic surgery is extremely well tolerated, safe and predictable. Increasingly, we are performing palliative procedures for women with advanced endometrial cancer with minimal negative impact. Robotic surgery is particularly well suited to high BMI patients; allowing surgical staging to be performed without undue difficulty or surgical compromise. This study demonstrates the lowest 30-day mortality $(0.14 \%)$ within our robotic cohort. Introduction of the Da Vinci robot in our Centre has led to a revolutionary change in practice with significant patient benefit. Many cases previously thought not fit for surgery at all, are now recommended robotic surgery.

Disclosures Anil Tailor: Proctor for Intuitive Surgical

Jayanta Chatterjee: paid-lectures on behalf of pharmaceutical companies

Agnieszka Michael: Educational-grants: Clovis, GSK, Ipsen, Novartis, Pfizer, and Tesaro

Simon Butler-Manuel: Proctor for Intuitive Surgical, Plasma Surgical \& Ethicon

\section{BRCA1/2 MUTATIONS PREDICT BETTER SURVIVAL IN HIGH-GRADE ENDOMETRIOID ENDOMETRIAL CANCER}

Yibo Dai, Jingyuan Wang, Luyang Zhao, Zhiqi Wang, Jianliu Wang. Peking University People's Hospital

\subsection{6/ijgc-2020-ESGO.60}

Introduction/Background Recent studies and clinical trials demonstrated the vital significance of BRCA mutational status in ovarian cancer treatment, but related evidence in endometrial cancer (EC) is still limited. This study aims to investigate the role of BRCA mutations in predicting EC patients' survival.

Methodology 510 eligible cases from the Cancer Genome Atlas database were retrospectively analysed. Clinicopathological characteristics of patients with different BRCA1/2 mutational status were compared. To analyse the survival influence of BRCA1/2 mutation, Kaplan-Meier survival analyses and Cox regressions were conducted. In order to control confounding bias between groups, propensity score matching method was used.

Results Among the eligible patients, 11 (2.2\%) harboured BRCA1 mutations, 43 (8.4\%) harboured BRCA2 mutations, and $36(7.1 \%)$ harboured both. Body mass index, rates of hypertension history, proportion of non-endometrioid histology and rates of positive peritoneal cytology were lower in BRCA1/2 mutant patients compared with the wild-type counterpart ( $\mathrm{p}=0.020,0.048,0.001$ and 0.012 , respectively). Patients with BRCA1/2 mutations showed longer overall (OS) and recurrence-free survival (RFS) (in Kaplan-Meier analyses, $\mathrm{p}<0.001$ and $\mathrm{p}=0.004$, respectively; in Cox regressions, $\mathrm{p}$ $=0.001$ and 0.007 , respectively). Further analyses indicated that the survival influence of BRCA1/2 mutations was only significant in high-grade endometrioid EC patients. Based on the cohorts generated after propensity score matching, in high-grade endometrioid EC patients, the influence of BRCA1/ 2 mutations remained significant on OS, but not on RFS ( $p$ $=0.003$ and 0.057 in Kaplan-Meier analyses, $\mathrm{p}=0.020$ and 0.071 in Cox regressions).

Conclusion BRCA1/2 mutations could predict better survival outcome in high-grade endometrioid EC patients, indicating the value of BRCA testing in EC clinical management.

Disclosures This work was supported by the National Natural Science Foundation of China (81972426 and 81874108), Special Projects for Strengthening Basic Research of Peking University (BMU2018JC005), National Key Technology R\&D Program of China (2019YFC1005200 and 2019YFC1005201).

The authors have no potential conflict of interest to disclose.

\section{WHICH DIETARY AND EVERYDAY LIFE HABITS AFFECT ENDOMETRIAL CANCER RECURRENCE? THE MACHINE GIVES THE ANSWER}

${ }^{1}$ Ofra Castro Wersäll, 'Zoia Razumova, ${ }^{2}$ Igor Govorov, ${ }^{3}$ Miriam Mints. ${ }^{1}$ Karolinska Institutet; Women's and Children's Health; ${ }^{2}$ Karolinska Institutet; Almazov National Medical Research Centre; Women's and Children's Health; ${ }^{3}$ Karolinska Institutet; Örebro University; Women's and Children's Health

\subsection{6/ijgc-2020-ESG0.61}

Introduction/Background The increased life expectancy and westernization of the lifestyle are considered the major contributors to a sustainable rise in endometrial cancer (EC) rates. The factors predicting EC recurrence include patient age and tumour characteristics, such as type, differentiation, and depth of invasion. At the same time, recent studies testify the impact of meal and exercises on the course of various diseases. What are the food preferences and activities that could influence the ultimate risk of EC relapse and death?

Methodology The study included 481 women who previously underwent a hysterectomy due to EC at Karolinska University Hospital. The participants filled an extensive questionnaire on their dietary habits and everyday routines. Related clinical data was obtained through the National e-health system. It resulted in a large dataset with more than 180 variables, which was processed using the Random Survival Forest (RSF) approach. The latter is applied to a right-censored data and uses a collection of decision trees to rank the variables by their importance for the occurrence of an event. The topranked variables were further investigated with the Cox proportional hazards model. Analyzes were performed using the RandomForestSRC package for Python.

Results The consumption of the fried potatoes significantly increased the risk of EC relapse and death $[\mathrm{HR}=8.62(2.22-$ 33.56), $\mathrm{p}=0.002 ; \mathrm{HR}=6.00(1.06-34.01), \mathrm{p}=0.043$, respectively), the latter persisted after adjustment for body mass index, age and smoking status. Besides, each additional serving of sweetened soda drinks increased the risk of death [3.262 (1.834-5.800), $\mathrm{p}=0.0001]$. In contrast, physical activity was beneficial with each additional Metabolic Equivalent per day decreasing the risk of death by $7.3 \%[\mathrm{HR}=0.927 \quad(0.892-$ 0.964), $\mathrm{p}<0.0001)$.

Conclusion We hypothesise that the fried potatoes' detrimental effect may be related to the acrylamide, which is formed in starch-rich foods under high-temperature conditions. It acts as 
a carcinogen and endocrine disruptor, causing the endometrial hyperplasia and EC in animal studies. Sweetened beverages cause a rise in insulin, which in turn inhibits sex-hormone binding protein. This results in higher levels of circulating free oestrogens. Also, insulin has mitogenic and anti-apoptotic properties, further inducing the endometrium proliferation. The favorable influence of regular physical activity on EC relapse and death is in accordance with previous studies, including recent meta-analysis.

Therefore, we encourage women treated for EC to consider reducing sweetened beverages and fried potatoes consumption and increasing physical activity.

Disclosures The authors have nothing to disclose.

\section{USE OF PREOPERATIVE AND INTRAOPERATIVE PARAMETERS FOR DECISION MAKING IN OVARIAN PRESERVATION IN ENDOMETRIAL ADENOCARCINOMA}

Halise Meltem Batur, Murat Gultekin, Mehmet Coskun Salman, Nejat Ozgul. Hacettepe University Faculty of Medicine; Department of Obstetrics and Gynaecology

\subsection{6/ijgc-2020-ESG0.62}

Introduction/Background Oophorectomy which is the integral part of surgery in endometrial adenocarcinoma leads to some adverse effects in premenopausal patients. Therefore, ovarian preservation concept has recently emerged especially in early stage disease. Several studies have shown that such approach does not adversely impact oncologic prognosis. This study aimed to retrospectively investigate the characteristics of endometrial adenocarcinoma patients with ovarian metastasis and to define criteria for ovarian preservation by using preoperative and intraoperative parameters.

Methodology Patients with endometrial adenocarcinoma who were operated at Hacettepe University Faculty of Medicine, Department of Obstetrics and Gynaecology were identified. The clinical and pathological characteristics of these patients were reviewed. Following univariate and multivariate analysis to determine factors associated with ovarian spread, different sets of criteria were analyzed to determine the subgroup of patients with no or negligible risk of ovarian metastasis.

Results The study group consisted of 725 patients and ovarian metastasis was detected in only $66(9.1 \%)$ of the patients. Univariate analysis showed tumor diameter, grade, histological type, myometrial invasion, peritoneal cytology, lymphovascular space invasion (LVSI), cervical invasion, omental and lymph node metastasis are significantly associated with ovarian metastasis while only LVSI, cervical invasion, omental and lymphatic involvement were significant on multivariate analysis. By using preoperative and intraoperative parameters only, no risk of ovarian metastasis was seen in patients of all ages with endometrioid tumor of any grade without myometrial invasion and risk was negligible $(0.7 \%)$ among 142 patients $(19.6 \%$ of study population) of any age with grade 1, endometrioid type tumor without deep myometrial invasion.

Conclusion Oophorectomy is not always necessary in endometrial adenocarcinoma. Preoperative and intraoperative uterusrelated factors may be used to define patients in whom ovarian preservation is safe similar to the approach used to determine surgical extent. Thus, ovaries may safely be preserved in almost $20 \%$ of patients with endometrial adenocarcinoma.

Disclosures No potential conflict of interest to declare.

\section{REAL-WORLD TREATMENT PATTERNS, HEALTHCARE RESOURCE USE, AND COSTS BY LINE OF THERAPY AMONG NEWLY DIAGNOSED ENDOMETRIAL CANCER PATIENTS}

${ }^{1}$ Chizoba Nwankwo, ${ }^{2}$ Ruchitbhai Shah, ${ }^{2}$ Nehemiah Kebede, ${ }^{2}$ Anuj Shah, ${ }^{2}$ Shelby Corman. ${ }^{1}$ Merck and Co., Inc; ${ }^{2}$ Pharmerit - an Open Health Company; Pharmerit International, Bethesda, MD, USA

\subsection{6/ijgc-2020-ESG0.63}

Introduction/Background Guidelines recommend surgery as primary therapy for endometrial cancer patients. Subsequent treatments can include radiation with/without systemic therapy depending on patients' prognosis. However, there is little data describing real-world treatment patterns and economic burden among newly diagnosed endometrial cancer patients. Therefore, this study aimed to assess real-world treatment patterns and healthcare costs by line of therapy (LOT) among newly diagnosed endometrial cancer patients.

Methodology Endometrial cancer patients newly diagnosed between January 2015 - June 2018 with continuous medical enrollment for 12 months prior and 6 months post diagnosis were identified in the Optum Clinformatics DataMart database. Treatments associated with endometrial cancer, including surgeries (bilateral salpingo-oophorectomy, hysterectomy and

Abstract 291 Table 1 Treatment patterns among newly diagnosed endometrial cancer patients by line of therapy

\begin{tabular}{|c|c|c|}
\hline Treatments & $\begin{array}{l}\text { First Line of } \\
\text { Therapy }\end{array}$ & $\begin{array}{l}\text { Second Line of } \\
\text { Therapy }\end{array}$ \\
\hline Surgery ${ }^{a}$ & $\mathrm{~N}=3,309$ & $\mathrm{~N}=47$ \\
\hline Hysterectomy & 3,274 (98.94\%) & $41(87.23 \%)$ \\
\hline Lymphadenectomy & $1,580(47.75 \%)$ & $20(42.55 \%)$ \\
\hline Bilateral salpingo-oophorectomy & $830(25.08 \%)$ & $11(23.40 \%)$ \\
\hline Radiation $^{2}$ & $\mathrm{~N}=884$ & $\mathrm{~N}=501$ \\
\hline Radiation only & $55(6.22 \%)$ & $330(65.87 \%)$ \\
\hline Radiation + systemic & $58(6.56 \%)$ & $163(32.53 \%)$ \\
\hline Radiation + surgery & $604(68.33 \%)$ & $4(0.8 \%)$ \\
\hline Radiation + surgery + systemic & $167(18.89 \%)$ & $4(0.8 \%)$ \\
\hline Systemic only a & $\mathrm{N}=152$ & $\mathrm{~N}=231$ \\
\hline Carboplatin & $49(32.24 \%)$ & $116(50.22 \%)$ \\
\hline Paclitaxel & $42(27.63 \%)$ & $100(43.29 \%)$ \\
\hline Megestrol acetate & $35(23.03 \%)$ & $12(5.19 \%)$ \\
\hline Other & $88(57.89 \%)$ & $144(62.34 \%)$ \\
\hline Systemic with surgery/radiation ${ }^{a}$ & $\mathrm{~N}=780$ & $\mathrm{~N}=184$ \\
\hline Carboplatin & $529(67.82 \%)$ & $80(43.48 \%)$ \\
\hline Paclitaxel & $507(65.0 \%)$ & $74(40.22 \%)$ \\
\hline Cisplatin & $55(7.05 \%)$ & $12(6.52 \%)$ \\
\hline Other & $320(41.0 \%)$ & $122(66.3 \%)$ \\
\hline
\end{tabular}

a The categories are not mutually exclusive as patients may have received a combination of these therapies. Radiation therapy includes external beam radiotherapy and brachytherapy.

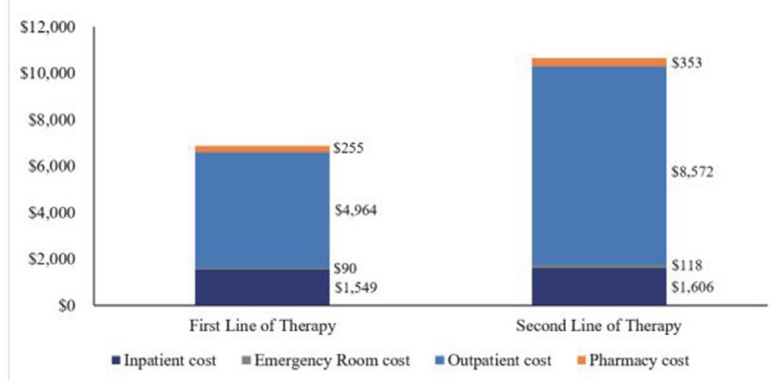

Abstract 291 Figure 1 Mean per patient per month healthcare costs by line of therapy among newly diagnosed endometrial cancer patients 